\title{
EDITORIAL
}

\section{Navigating the regulatory road to approval for cancer gene therapies}

Cancer Gene Therapy (2015) 22, 553. doi:10.1038/cgt.2015.62

On 27 October 2015 the US Food and Drug Administration announced that it had approved Imlygic (talimogene laherparepvec), an oncolytic virus, for the treatment of melanoma that cannot be removed by surgery. The genetically modified live herpes virus is injected intralesionally into tumors and can be administered repeatedly. This approval marks a major milestone in the development of viral gene therapy and hopefully heralds the start of a new era in the translation of vector-based therapies for patients with cancer.

In this issue of Cancer Gene Therapy, Drs Husain, Han, Au, Shannon and Puri of the FDA present a timely review of the important considerations that go into the evaluation of gene therapy strategies being considered for regulatory approval. This primer presents not only an overview of the various strategies and vector systems, it also outlines chemistry, manufacturing and control considerations, pre-clinical considerations, clinical considerations and proper communication strategies with the FDA. I am grateful to Dr Puri and his team for this contribution, and I consider it a must read for anyone developing gene therapies for patients with cancer.

While hurdles still remain, recent advances in oncolytic viral therapies, adoptive cell transfer of genetically modified immune cells and other vector platforms give us reason to be very optimistic. The road forward is clearer than at any other time for the field. It is my hope that this review and overview will be of great interest and use to the readers of Cancer Gene Therapy.

SK Libutti Montefiore Medical Center and Albert Einstein College of Medicine, Greene Medical Arts Pavilion, Bronx, NY, USA E-mail: slibutti@montefiore.org 